DEMONSTRATIO MATHEMATICA

Vol. XXVIII No $2 \quad 1995$

Izydor Dziubiński, Jerzy Połubiński

\title{
SOME RESULTS OF ANALYSIS \\ IN THE NORMED ALGEBRAS
}

This paper concerns some problems of analysis in the normed (complex or real) algebra $X$.

The main tool in the investigations of the functions in complex commutative Banach algebras with unity is the Gelfand theory of maximal ideals (see [1], [3]). Developing this idea E. R. Lorch in [3] put forward the question when in algebra $X$, the following conditions are equivalent:

(1) differentiability of the function (in the sense defined by Lorch),

(2) vanishability of the line integral of the function along the closed curve,

(3) power series expansibility of the function.

This paper includes, in particular, some proposal of the method of solution of this problem without using Gelfand theory.

The presented theory is based on two ideas:

- derivative of the function and, consequently, the primitive function as a map from $X$ to $X$, (chapter 2);

- surrounding subsets of the $X$ by closed curves, (chapter 5 ).

Main results of this paper are parallel to the Cauchy-Riemann equations, Cauchy-integral theorem, Cauchy-integral formula, Taylor and Laurent series expansions, Morera and Liouville theorems.

\section{Introduction}

Let $X$ be a normed algebra (commutative or non-commutative) over the field $K(\mathbb{C}$ or $\mathbb{R})$. We will use the following denotations: by $\mathbb{N}$-set of all natural numbers, $\mathbb{N}_{0}:=\mathbb{N} \cup\{0\}$. For $x \in X$ and $r>0$ by $B(x, r)$ we will denote as usual the open ball with the centre $x$ and the radius $r$. By $\delta_{x} f(h)$ we will denote directional derivative of the function $f$ at a point $x$ in the direction $h ; f^{\prime}(x)$-Frechet's derivative at a point $x$. For $x_{0}, x_{1} \in X$, 
$x_{0} \neq x_{1}$ by $\left(x_{0}, x_{1}\right)$ we will denote the segment $\left\{x_{0}+t\left(x_{1}-x_{0}\right): t \in(0,1)\right\}$. Analogously we define the segments $\left(x_{0}, x_{1}\right\rangle,\left\langle x_{0}, x_{1}\right)$ and $\left\langle x_{0}, x_{1}\right\rangle$.

Now let us define

$$
\begin{aligned}
L & :=\left\{x \in X: \bigwedge_{0 \neq y \in X} x y \neq 0\right\}, \quad \text { for } a \in \mathbb{R}_{+} \\
L^{a} & :=\left\{x \in X: \bigwedge_{y \in X} a\|X\|\|y\| \leq\|x y\|\right\} \text { and } L_{0}^{a}:=L^{a} \backslash\{0\} .
\end{aligned}
$$

From the definitions there follows immediately

LEMMA 1.1. For any $a>0$ the set $L^{a}$ is closed and $L_{0}^{a} \subset L$; for $a>1$ $L_{0}^{a}=\emptyset$ and if $0<a_{1} \leq a_{2}$, then $L^{a_{2}} \subset L^{a_{1}}$.

We introduce the following notation: $L^{*}=\bigcup_{a>0} L^{a}, L_{0}^{*}=L^{*} \backslash\{0\}$,

$$
a_{l}=\sup \left\{a: L_{0}^{a} \neq \emptyset\right\} \quad \text { and for } \quad x \in L_{0}^{*} \quad P_{x}=\sup \left\{a: x \in L_{0}^{a}\right\} \text {. }
$$

Later on we will often consider the sets $D \subset X$ satisfying the following property:

$$
\text { if } x \in D \quad \text { and } \quad 0 \neq t \in K \text {, then } t x \in D \text {. }
$$

It is clear that if the set $D$ and the sets of the family $\left\{D_{s}\right\}$ have property (*), then $\bigcup D_{s}, \cap D_{s}$ and $X \backslash D$ also possess (*).

It is also clear that $L_{0}^{*} \subset L$ and the sets $L^{a}, L_{0}^{a}, L^{*}$ and $L_{0}^{*}$ have property $(*)$.

Lemma 1.2. (a) If $x_{0} \in L_{0}^{a}$ for some a $>0$, then for every $\varepsilon>0$ there exists such a number $b_{\varepsilon}>0$ that $B_{\varepsilon}:=B\left(x_{0}, \frac{a\left\|x_{0}\right\|}{1+\varepsilon}\right) \subset L_{0}^{b_{c}}$.

(b) If $x \in L_{0}^{a}$ and $b \in(0, a)$, then $B\left(x, \frac{a-b}{a+b}\|x\|\right) \subset L_{0}^{b}$.

P roof. Let us take $\varepsilon>0$ and $x_{0} \in B_{\varepsilon}$. By $1.1 a \leq 1$, hence

$$
\left\|x_{1}-x_{0}\right\|<\frac{a\left\|x_{0}\right\|}{1+\varepsilon}<\left\|x_{0}\right\| \text {. }
$$

Since $x_{0} \in L_{0}^{a}$, so for $x, y \in X$

$$
a\left\|x_{0}\right\|\|y\| \leq\left\|x_{0} y\right\| \leq\left\|\left(x_{0}-x\right) y\right\|+\|x y\| \leq\left\|x-x_{0}\right\|\|y\|+\|x y\| .
$$

Therefore for $x \neq 0$

$$
b_{x}\|x\|\|y\| \leq\|x y\|, \quad \text { where } b_{x}=\frac{a\left\|x_{0}\right\|-\left\|x-x_{0}\right\|}{\|x\|} .
$$

It follows from (1) that $x_{1} \neq 0$ and

$$
b_{x_{1}}>\frac{a\left\|x_{0}\right\|}{\left\|x_{1}\right\|}-\frac{a\left\|x_{0}\right\|}{(1+\varepsilon)\left\|x_{1}\right\|}=\frac{a\left\|x_{0}\right\|}{\left\|x_{1}\right\|} \cdot \frac{\varepsilon}{1+\varepsilon} .
$$


Since $\left\|x_{1}\right\| \leq\left\|x_{1}-x_{0}\right\|+\left\|x_{0}\right\|$, then from (1) we obtain $b_{x_{1}}>\frac{a \varepsilon}{(1+\varepsilon)+a}:=$ $b_{\varepsilon}>0$. Hence and from (2) it follows that $x_{1} \in L_{0}^{b_{e}}$. For the proof of (b) it is enough to put $\varepsilon=\frac{b(1+a)}{a-b}$.

1.3 Corollary. If $x \in L_{0}^{a}$, then $B(x, a\|x\|) \subset L_{0}^{*}$.

Thus, if $x \in L_{0}^{*}$, then $B\left(x, P_{x}\|x\|\right) \subset L_{0}^{*}$. From 1.2 and 1.3 we get the following

1.4 TheOREM. The set $L_{0}^{*}$ is open. If $a_{n}>0$ for $n \in \mathbb{N}$ and $\lim a_{n}=0$, then $L_{0}^{*}=\bigcup_{n}$ Int $L_{0}^{a_{n}}=\bigcup_{a>0} \operatorname{Int} L_{0}^{a}$.

An element of the set $X \backslash L^{*}$ will be called a divisor of zero. Element $x \in X, x \neq 0$ will be called a topological divisor of zero if there exists such a sequence $\left(x_{n}\right)$ in $X$ that $\left\|x_{n}\right\|=1$ for $n \in \mathbb{N}$, and $\lim _{n \rightarrow \infty} x x_{n}=0$.

From the definitions of the sets: $L_{0}^{*}, L^{*}$ and $L$ there follows

Lemma 1.5. No topological divisor of zero lies in $L_{0}^{*}$ and every element of the set $X \backslash L^{*}$ is a topological divisor of zero. $L \backslash L^{*}$ is the set of topological divisors of zero which are not the divisors of zero.

Indeed, if $x \in L_{0}^{*}$ and $\left\|x_{n}\right\|=1$, then $\left\|x x_{n}\right\| \geq P_{x}\|x\|>0 . x \in X \backslash L^{*}$ implies that $x \notin L^{1 / n}$ for any $n \in \mathbb{N}$. Therefore there exists a sequence $\left(y_{n}\right) \subset X$ such that $\left\|y_{n}\right\|=1$ and $\left\|x y_{n}\right\|<\|x\| / n$. Hence $\lim _{n \rightarrow \infty} x y_{n}=0$. The last part of the statement follows from the definition of the set $L$.

1.6 ThEOREM. If $\operatorname{dim} X<\infty$, then $L_{0}^{*}=L$.

P roof. Let $x \in L \backslash L_{0}^{*}$ and $S_{1}=\{x \in X:\|x\|=1\}$. Then there exists a sequence $\left(x_{n}\right) \subset S_{1}$ such that $\lim x x_{n}=0$. The compactness of $S_{1}$ implies the existence of subsequence $\left(x_{n_{k}}\right)$ convergent to $x_{0} \in S_{1}$. Then $0=\lim x x_{n_{k}}=x x_{0}$, thus $x \notin L$.

1.7 Examples. (a) Fix $n \in \mathbb{N}$. Let $X$ denote an algebra in $\mathbb{R}^{n}$ over field $\mathbb{R}$. Then there exist two-linear mappings $a_{k}: X^{2} \rightarrow \mathbb{R}, k=1,2, \ldots, n$, such that for any $x=\left(x_{i}\right)_{i=1}^{n}, y=\left(y_{j}\right)_{j=1}^{n} \in X x y=\left(a_{k}(x, y)\right)_{k=1}^{n}=$ $\left(\sum_{j=1}^{n} b_{k}^{j}(x) y_{j}\right)_{k=1}^{n}$, where $b_{k}^{j}(x)=\sum_{i=1}^{n} a_{k}^{i j} x_{i}$ and $a_{k}^{i j} \in \mathbb{R}$. Let $B(x)=$ $\operatorname{det}\left[b_{k}^{j}(x)\right]_{j, k \leq n}$. It is obvious that

$$
B(x)=B\left(x_{1}, x_{2}, \ldots, x_{n}\right)=\sum_{i_{1}, \ldots, i_{n}=1}^{n} x_{i_{1}} x_{i_{2}} \ldots x_{i_{n}} \operatorname{det}\left[a_{j}^{i_{k} k}\right]_{j, k \leq n}
$$

and $L=\{x \in X: B(x) \neq 0\}$. From $1.6 L=L_{0}^{*} . B\left(x_{1}, \ldots, x_{n}\right)$ is a homogeneous polynomial, and we can factorize it, explicitly up to a constant coefficient, as the prime homogeneous polynomials:

$$
B\left(x_{1}, \ldots, x_{n}\right)=F_{1}^{d_{1}}\left(x_{1}, \ldots, x_{n}\right) \ldots F_{m}^{d_{m}}\left(x_{1}, \ldots, x_{n}\right),
$$


where $d_{j}=\operatorname{deg} F_{j}^{d_{j}}$. Therefore $X \backslash L_{0}^{*}$ is the union of the hypersurfaces defined by the equations $F_{j}^{d_{j}}\left(x_{1}, \ldots, x_{n}\right)=0$. The analogous result will be obtained taking $X=\mathbb{C}^{n}$.

(b) Let $X$ be a commutative algebra $\mathcal{C}(0,1)$ of real functions continuous on the interval $I=\langle 0,1\rangle$, over field $\mathbb{R}$. Taking $N(x)=\{t \in I: x(t)=0\}$ for $x \in X$, we conclude that $L$ is the set of those $x \in X$ for which $N(x)$ is a boundary set. For $a>0, L^{a}$ is the set of $x \in X$ of the form $x=s x_{0}$, where $s \in \mathbb{R}, x_{0} \in X,\left\|x_{0}\right\|=1$ and $\min _{I}|x(t)| \geq a$. Consequently, $L=\{x \in X:$ $\left.\min _{I}|x(t)|>0\right\}$.

\section{Derivative}

Let $L_{0}^{*} \neq \emptyset$, and let us fix any $c \in L_{0}^{*},\|c\|=1$. Element $y \in X$ will be called lc-inverse (left-hand-c-inverse) of $x \in X$ if $x y=c$. The set of point $x \in X$ for which there exists an lc-inverse element will be denoted by $r_{l}(c)$.

Let $L_{c}^{*}:=L_{0}^{*} \cap \operatorname{Int} r_{l}(c) \neq \emptyset$ and for $a>0 L_{c}^{a}:=L_{0}^{a} \cap \operatorname{Int} r_{l}(c)$.

The sets $r_{l}(c), L_{c}^{*}$ and $L_{c}^{a}$ have property (*). For every $x \in L_{c}^{*}$ there exists only one lc-inverse element $A_{c}^{l}(x)$. Therefore we have defined a mapping $A_{c}^{l}$ on $L_{c}^{*}$. For every $x \in L_{c}^{*}$ we get obviously the inequality

$$
\frac{1}{\|x\|} \leq\left\|A_{c}^{l}(x)\right\| \leq \frac{1}{P_{x}\|x\|} \text {. }
$$

Lemma 2.1. For any $a>0$ there exists $b_{a}>0$ such that

$$
A_{c}^{l}\left(L_{c}^{a}\right) \subset L_{0}^{b_{a}}
$$

Thus, in particular, $A_{c}^{l}\left(L_{c}^{*}\right) \subset L_{0}^{*}$.

P r o of. If $A_{c}^{l}$ does not have property (1), then for any $a>0$ there exists a sequence $\left(x_{n}\right) \subset L_{c}^{a},\left\|x_{n}\right\|=1$, such that $y_{n}=A_{c}^{l}\left(x_{n}\right) \in X \backslash L^{a / n}$ for $n \in \mathbb{N}$. Thus there exists $z_{n} \in X$ such that $\left\|z_{n}\right\|=1$ and $\left\|y_{n} z_{n}\right\|<\frac{a}{n}\left\|y_{n}\right\| \leq \frac{1}{n}$. Hence $\left\|c z_{n}\right\|=\left\|x_{n}\left(y_{n} z_{n}\right)\right\| \leq \frac{1}{n} \rightarrow 0$, hence which implies that $c$ is a topological divisor of zero (see 1.5) and, consequently, $c \notin L_{0}^{*}$.

Let us take an arbitrary $x \in X, r>0$ and a function $f$ defined on the set $K(x, r)=\left(B(x, r) \cap\left(L_{c}^{*}+x\right)\right) \cup\{x\}$ with values in $X$.

If for an arbitrary $a \in\left(0, a_{l}\right)$ there exist the limits

$$
\lim _{L_{c}^{a} \ni h \rightarrow 0} A_{c}^{l}(h)(f(x+h)-f(x))
$$

then by 1.1 these limits are equal for all $a$. Their common value will be denoted by $D_{l c} f(x)$ and called an lc-derivative of the function $f$ at the point $x$. 
If $f$ has the lc-derivative at each point of the set $M \subset X$, then the mapping $D_{l c}: M \ni x \mapsto D_{l c} f(x)$ will be called the lc-derivative of the function $f$ on $M$.

For $n \in \mathbb{N}_{0}$ we define the lc-derivative of order $n$ of the function $f$ as follows: $D_{l c}^{0} f=f, D_{l c}^{n} f=D_{l c}\left(D_{l c}^{n-1} f\right)$ for $n>0$.

2.2 Theorem. For every $x \in X$

(a) if there exists $D_{l c} f(x)$ and for some $h \in L_{c}^{*}$ there exists $\delta_{x} f(h)$, then $D_{l c} f(x)=A_{c}^{l}(h) \delta_{x} f(h)$;

(b) if there exists $D_{l c} f(x)$ and $c$ is the unity in $X$ (i.e. $c z=z c=z$ for every $z \in X)$, then for every $h \in L_{c}^{*}$ there exists $\delta_{x} f(h)$ and $D_{l c} f(x)=$ $A_{c}^{l}(h) \delta_{x} f(h)$.

P roof. Let $0 \neq t \in K, h \in L_{c}^{*}$ and $I(t):=t^{-1}(f(x+t h)-f(x))$. Therefore $t h \in L_{c}^{P_{h}}$ and $D_{l c} f(x)=\lim _{t \rightarrow 0} A_{c}^{l}(t h)(f(x+t h)-f(x))=$ $\lim _{t \rightarrow 0} A_{c}^{l}(h) I(t)=A_{c}^{l}(h) \delta_{x} f(h)$. If $h \in L_{c}^{*}$, then for some $a>0 A_{c}^{l}(h):=$ $z \in L_{0}^{a}$. As $c$ is the unity, so $z h=c$, and

$$
0=\lim _{t \rightarrow 0}\left\|z I(t)-z h D_{l c} f(x)\right\| \geq a\|z\| \lim _{t \rightarrow 0}\left\|I(t)-h D_{l c} f(x)\right\| .
$$

2.3 ThEOREM. If the function $f$ has the Fréchet derivative $f_{x}^{\prime}$ at some point $x$ and

$$
A_{c}^{\prime}(h) f_{x}^{\prime}(h):=d
$$

does not depend on $h \in L_{c}^{*}$, then $f$ has the lc-derivative at the point $x$ which is equal to $d$, and $\left\|D_{l c} f(x)\right\| \leq a_{l}^{-1}\left\|f_{x}^{\prime}\right\|$.

P roof. Let $R(x, h)=f(x+h)-f(x)-\delta_{x} f(h)$. From (1) for $h \in L_{c}^{*}$ we obtain

$$
A_{c}^{l}(h)(f(x+h)-f(x))-d=A_{c}^{l}(h) R(x, h) .
$$

Let us suppose that $h \in L_{c}^{a}$ for any fixed $a \in\left(0, a_{l}\right)$. From (2.1) we have:

$$
\left\|A_{c}^{l}(h) R(x, h)\right\| \leq a^{-1}\|h\|^{-1}\|R(x, h)\| \underset{h \rightarrow 0}{\longrightarrow} 0 .
$$

Thus, it follows from (2) that $d=D_{l c} f(x)$ and $\left\|D_{l c} f(x)\right\|=\left\|A_{c}^{l}(h) \delta_{x} f(h)\right\|$ $\leq\left\|f_{x}^{\prime}\right\|\|h\| a^{-1}\|h\|^{-1}$. Hence $\left\|D_{l c} f(x)\right\| \leq a_{l}^{-1}\left\|f_{x}^{\prime}\right\|$.

The set $L_{c}^{*}$ is open, thus includes a ball. Therefore, there exists a Hamelbasis $H=\left\{h_{s}\right\}$ of the space $X$ such that $H \subset L_{c}^{*}$. Let us fix such the basis. From 2.2 and 2.3 there follows

2.4 THEOREM. (a) If the function $f$ has the lc-derivative at the point $x \in X$ and the directional derivative in direction $h_{s}, h_{t} \in H$, then

$$
A_{c}^{l}\left(h_{s}\right) \delta_{x} f\left(h_{s}\right)=A_{c}^{l}\left(h_{t}\right) \delta_{x} f\left(h_{t}\right):=d
$$

and $d=D_{l c} f(x)$. 
(b) If $c$ is the unity of an algebra $X$ (or $X$ is a commutative algebra) and if the function $f$ has the Fréchet derivative at the point $x$, and for any $h_{s}, h_{t} \in H$ the equations (2.2) hold, then $f$ has the lc-derivative equals $d$ at the point $x$.

The equations (2.2) are equivalent to Cauchy-Riemann equations.

If the function $f$ has the lc-derivative at the point $x$, then it does not have to be continuous there. However, we have the following

Lemma 2.5. If $f$ has the lc-derivative at the point $x$, then for any $a \in$ $\left(0, a_{l}\right)$ there exist such positive numbers $r_{a}$ and $G_{a}$ that for $h \in B\left(0, r_{a}\right) \cap L_{c}^{a}$ $\|f(x+h)-f(x)\| \leq G_{a}\|h\|$ is satisfied.

Proof. For $a \in\left(0, a_{l}\right)$ let us choose $b_{a}$ such that for $h \in L_{c}^{a} \quad A_{c}^{l}(h) \in L_{0}^{b_{a}}$ (see 2.1). Applying (2.1) we get : $\|f(x+h)-f(x)\| \leq b_{a}^{-1} \| A_{c}^{l}(h)(f(x+h)-$ $f(x))\|\| h \|$. The existence of the lc-derivative of the function $f$ at the point $x$ implies that for some $r_{a}>0$ and $h \in B\left(0, r_{a}\right) \cap L_{c}^{a}$ we obtain:

$$
\begin{aligned}
1 & \geq\left\|A_{c}^{l}(h)(f(x+h)-f(x))-D_{l c} f(x)\right\| \\
& \geq\left\|A_{c}^{l}(h)(f(x+h)-f(x))\right\|-\left\|D_{l c} f(x)\right\| .
\end{aligned}
$$

We take $G_{a}=b_{a}^{-1}\left(\left\|D_{l c} f(x)\right\|+1\right)$; the proof is completed.

We can prove that if $X$ is commutative and if $f$ has the lc-derivative bounded in the domain $M \subset X$, then $f$ is continuous on $M$.

A function $f$ will be called strong lc-differentiable of order $n \in \mathbb{N}$ in the set $M \subset X$, if it has the lc-derivative of order $n$ and the Fréchet derivative of order $n$ in $M$. The set of such functions will be denoted by $\mathcal{H}_{l c}^{n}(M), \mathcal{H}_{l c}(M)$ means $\mathcal{H}_{l e}^{1}(M)$ and $\mathcal{H}_{l c}^{\infty}(M):=\bigcup_{n=1}^{\infty} \mathcal{H}_{l c}^{n}(M)$.

LEMMA 2.6. If the set $M \subset X$ possesses the property $(*)$ and contains a ball, then there exists such a number $G>0$ that for any $z_{1}, z_{2} \in X, z_{1} \neq z_{2}$ and for some $y \in\left(z_{1}+M\right) \cap\left(z_{2}+M\right)$ the inequalities $\left\|z_{i}-y\right\| \leq G\left\|z_{1}-z_{2}\right\|$, $i=1,2$ hold.

P r o of. 1. Let $z_{2}-z_{1} \in M$, then $\left(z_{1}, z_{2}\right) \subset z_{1}+M$. Then taking $y=$ $\frac{1}{2}\left(z_{1}+z_{2}\right)$ we get $y \in\left(z_{1}+M\right) \cap\left(z_{2}+M\right)$ and $\left\|z_{i}-y\right\|=\frac{1}{2}\left\|z_{1}-z_{2}\right\|$. Thus we can take $G=\frac{1}{2}$. Similarly, when $z_{1}-z_{2} \in M$.

2. Let us now suppose that $z_{1}-z_{2} \notin M$. For some $x_{0} \in M$ and $r>0$ $B\left(x_{0}, r\right) \subset M$. Let $t_{0}:=\left\|z_{1}-z_{2}\right\| r^{-1}$, then $B\left(t_{0} x_{0}, t_{0} r\right) \subset M$. Therefore $B_{i}:=B\left(z_{i}+t_{0} x_{0}, t_{0} r\right) \subset z_{i}+M$ for $i=1,2$. Since $\left\|\left(z_{1}+t_{0} x_{0}\right)-\left(z_{2}+t_{0} x_{0}\right)\right\|=$ $\left\|z_{1}-z_{2}\right\|=t_{0} r<2 t_{0} r$, thus there exists $y \in B_{1} \cap B_{2}$. Then for $i=1,2$ we derive that:

$$
\begin{aligned}
\left\|y-z_{i}\right\| & \leq\left\|y-\left(z_{i}+t_{0} x_{0}\right)\right\|+\left\|z_{i}+t_{0} x_{0}-z_{i}\right\| \leq\left\|z_{1}-z_{2}\right\|+t_{0}\left\|x_{0}\right\| \\
& =\left(1+\left\|x_{0}\right\| r^{-1}\right)\left\|z_{1}-z_{2}\right\| .
\end{aligned}
$$


Concluding, we can take $G=1+\left\|x_{0}\right\| r^{-1}$.

2.7 ThEOREM. If $D_{l c} f=0$ in a certain domain $M \subset X$, then $f$ is constant on $M$.

The proof is preceded by two lemmas.

Lemma 1. If $D_{l c} f(x)=0$ for certain $x \in X$, then $\delta_{x} f(h)=0$ for any $h \in L_{c}^{*}$.

Indeed, let us fix any $h \in L_{c}^{*} . t \neq 0$ implies that $t h \in L_{c}^{P_{h}}$. Thus, $0=$ $D_{l c} f(x)=\lim _{t \rightarrow 0} A_{c}^{l}(h) I(t)$, where $I(t)=t^{-1}(f(x+t h)-f(x))$. From 2.1 it follows that $z_{h}:=A_{c}^{l}(h) \in L_{0}^{b}$ for some $b>0$. Hence $0=\lim _{t \rightarrow 0}\left\|z_{h} I(t)\right\| \geq$ $b\left\|z_{h}\right\| \lim _{t \rightarrow 0}\|I(t)\|$, therefore $\delta_{x} f(h)=0$.

LemMA 2. Let $B \subset X$ be a ball and $x_{0} \in B$. Then if $D_{l c} f(x)=0$ for every $x \in K_{x_{0}}:=\left(B \cap\left(x_{0}+L_{c}^{*}\right)\right) \cup\left\{x_{0}\right\}$, then $f$ is constant on $K_{x_{0}}$.

Proof. Let us take $x_{1} \in K_{x_{0}}, x_{1} \neq x_{0}$. Then $x_{1}-x_{0} \in L_{c}^{*}$ and $\left\langle x_{0}, x_{1}\right\rangle \subset$ $K_{x_{0}}$. Since $B$ is open there exists the a segment $\left(\bar{x}_{0}, \bar{x}_{1}\right) \subset K_{x_{0}}$ such that $\left\langle x_{0}, x_{1}\right\rangle \subset\left(\bar{x}_{0}, \bar{x}_{1}\right) \subset K_{x_{0}}$. From Lemma 1 we have that $\delta_{x} f(h)=0$ for $x \in K_{x_{0}}$ and $h \in L_{c}^{*}$. In particular $\delta_{x} f\left(x_{1}-x_{0}\right)=0$ for $x \in\left(\bar{x}_{0}, \bar{x}_{1}\right)$. Hence it follows that $f$ is constant on $\left(\bar{x}_{o}, \bar{x}_{1}\right)$, thus $f\left(x_{0}\right)=f\left(x_{1}\right)$.

P roof of 2.7. Firstly let us suppose that $M$ is a ball and let us choose arbitrary $x_{0}, x_{1} \in M$. Then $\left\langle x_{0}, x_{1}\right\rangle \subset M$. Let $d:=\operatorname{dist}\left(\left\langle x_{0}, x_{1}\right\rangle, \operatorname{Fr} M\right)$ and for $x \in M K_{x}=\left(M \cap\left(x+L_{c}^{*}\right)\right) \cup\{x\}$.

Let us choose from the segment $\left\langle x_{0}, x_{1}\right\rangle$ a sequence of points $z_{0}=x_{0}$, $z_{1}, \ldots, z_{n}=x_{1}$ and $y_{i} \in\left(z_{i}+L_{c}^{*}\right) \cap\left(z_{i+1}+L_{c}^{*}\right)$ such that $\left\|z_{i}-y_{i}\right\| \leq$ $G\left\|z_{i+1}-z_{i}\right\|$ and $G\left\|z_{i+1}-z_{i}\right\|<d$ for $i=0,1, \ldots, n-1$, and some $G>0$ (see 2.6). Then $y_{i} \in M$ which gives that $y_{i} \in K_{z_{i}} \cap K_{z_{i+1}}$.

From Lemma 2 it follows that for every $x \in M f$ is constantly equal to $f(x)$ on $K_{x}$, consequently

$$
f\left(x_{0}\right)=f\left(y_{0}\right)=f\left(z_{1}\right)=f\left(y_{1}\right)=\ldots=f\left(y_{n-1}\right)=f\left(z_{n}\right)=f\left(x_{1}\right) .
$$

Now let $M$ be an arbitrary domain. For any $x_{0}, x_{1} \in M$ there exists a complete sequence of balls $B_{1}, B_{2}, \ldots, B_{n}$ contained in $M$ that $x_{0} \in B_{1}$, $x_{1} \in B_{n}$ and $B_{i} \cap B_{i+1} \neq \emptyset$ for $i=1,2, \ldots, n-1$. As in each of these balls $f$ is constant, $f\left(x_{0}\right)=f\left(x_{1}\right)$.

Let $M \subset X$ and let $f$ be a function from $M$ to $X$. Then a function $F$ from $M$ to $X$ is called lc-primitive of $f$ on $M$ if $D_{l c} F(x)=f(x)$ for $x \in M$.

From 2.7, if the functions $F_{1}, F_{2}$ are lc-primitive of $f$ on the domain $M \subset X$, then $F_{1}-F_{2}$ is the constant function on $M$. 
2.8 ExAmples. (a) For every linear mapping $f: X \rightarrow X$, if there exists $D_{l c} f\left(x_{0}\right):=d$ at some $x_{0} \in X$, then $D_{l c} f(x)=d$ for any $x \in X$, since $f \in \mathcal{H}_{l c}(X)$.

(b) If $X$ is an algebra $\mathbb{C}$ with unity $e=1$, then for every $a \in(0,1\rangle$ we have $L_{0}^{a}=L_{0}^{*}=r_{l}(e)=\mathbb{C} \backslash\{0\}$. The le-derivative of the function agrees with the classical derivative. A class $\mathcal{H}_{\mathrm{le}}(D)$ for $D \subset \mathbb{C}$ is the class of holomorphic functions on $D$.

(c) Let $X$ be an algebra in $\mathbb{R}^{2}$ over field $\mathbb{R}$, where for $z_{1}=\left(x_{1}, y_{1}\right)$, $z_{2}=\left(x_{2}, y_{2}\right) \in X, z_{1} z_{2}:=\left(x_{1} x_{2}+y_{1} y_{2}, x_{1} y_{2}+x_{2} y_{1}\right) . X$ is a commutative algebra (complex hyperbolic numbers, see [5]) with unity $e=(1,0) ; L=$ $L_{e}^{*}=\left\{(x, y) \in \mathbb{R}^{2}: x^{2} \neq y^{2}\right\}$. For a function $f: z=(x, y) \mapsto(u(z), v(z))$ of $X$ to $X$, equations (2.2) can be written in the form : $u_{x}^{\prime}=v_{y}^{\prime}, u_{y}^{\prime}=v_{y}^{\prime}$.

(d) Let $X$ be an algebra in $\mathbb{C}^{2}$ over the field $\mathbb{C}$, where for $z_{j}=\left(x_{j}, y_{j}\right)$, $j=1,2, z_{1} z_{2}=\left(x_{1} x_{2}-y_{1} y_{2}, x_{1} y_{2}+x_{2} y_{1}\right) . X$ is a commutative algebra with unity $e=(1,0), L=L_{0}^{*}=\left\{(x, y) \in \mathbb{C}^{2}: x^{2}+y^{2} \neq 0\right\}$. For a function $f: z=(x, y) \mapsto(u(z), v(z))$ of $X$ to $X$ equations $(2,2)$ can be written in the form: $u_{x}^{\prime}=v_{y}^{\prime}, u_{y}^{\prime}=-v_{x}^{\prime}$.

(e) In the example $1.7(b)$ we considered the algebra $X=\mathcal{C}(0,1)$ with unity $e$. By a symbol $(x(t))$ let us denote a continuous function $t \mapsto x(t)$ for $t \in\langle 0,1\rangle$. Notice that a mapping $f: x \mapsto\left(\int_{0}^{t} x(s) d s\right)$ from $X$ to $X$ has not the le-derivative in any point. Indeed, this mapping is linear, for $x \in X$ and for $h \in L_{e}^{*}$ we have $\delta_{x} f(h)=\left(\int_{0}^{t} h(s) d s\right)$ and $A_{e}^{l}(h) \delta_{x} f(h)=$ $\left(h(t)^{-1} \int_{0}^{t} h(s) d s\right.$ ) depends on $h$ (see 2.2). In this algebra all functions $p_{n}$ : $x \mapsto x^{n}$ for $n \in \mathbb{N}_{0}$ have the le-derivative and $D_{\mathrm{le}} p_{n}=n p_{n-1}$ for $n \in \mathbb{N}$.

\section{Integral and primitive function}

By a curve in an algebra $X$ we understand a continuous mapping $\gamma$ of the interval $\langle\alpha, \beta\rangle \subset \mathbb{R}$ in $X$. Then $\langle\alpha, \beta\rangle$ is called the parameter interval of the curve $\gamma, \gamma(\alpha)$ - the beginning, $\gamma(\beta)$ - the end of the curve $\gamma$. We also will use the notation: $\gamma^{*}=\{\gamma(t): t \in\langle\alpha, \beta\rangle\}$. A curve $\gamma$ is called regular, or a path, if it is of class $C_{1}$ in the parameter interval. A point $x$ of a curve $\gamma$ is a said to be $k$-valent $(k \in \mathbb{N})$, if there exist exactly $k$ different numbers $t_{1}, t_{2}, \ldots, t_{k}$ from the interval parameter $\langle\alpha, \beta\rangle$ such that $x=\gamma\left(t_{1}\right)=\gamma\left(t_{2}\right)=\ldots=\gamma\left(t_{k}\right)$. By the length of the path $\gamma$ we mean the number $|\gamma|=\int_{\alpha}^{\beta}\left\|\gamma^{\prime}(t)\right\| d t$.

Let $\gamma:\langle\alpha, \beta\rangle \rightarrow X$ be a path in $X$ and and let $f$ be a continuous function on $\gamma^{*}$ in $X$. The $l$-integral of the function $f$ over the path $\gamma$ we define as follows: $\int_{\gamma} f(z) d z=\int_{\alpha}^{\beta} \gamma^{\prime}(t) f(\gamma(t)) d t$.

The last integral is the integral in the Riemann sense. Its existence follows from the continuity of $f$. (See e.g. [4]) 
Let $L_{0}^{*} \neq \emptyset$ and let us fix any $c \in L_{0}^{*},\|c\|=1$.

A path $\gamma$ is said to be correlated with $L_{c}^{a}$ at some point $t_{0} \in\langle\alpha, \beta\rangle$ if $\gamma(t)-\gamma\left(t_{0}\right) \in L_{c}^{a} \cup\{0\}$ for $t$ taken from some neighbourhood of the point $t_{0}$. If $\gamma$ is correlated with $L_{c}^{a}$ at every point, at most except the finite number of the elements of interval $\langle\alpha, \beta\rangle$, then we will say that $\gamma$ is correlated with $L_{c}^{a}$.

If $\gamma$ is correlated with $L_{c}^{a}$ and $b \in(0, a)$, then $\gamma$ is correlated with $L_{c}^{b}$.

We can formulate the following definition:

A path $\gamma$ will be called correlated with $L_{c}^{*}$ (at the point $t_{0}$ ) if there exists $a>0$ such that $\gamma$ is correlated with $L_{c}^{a}$ (at the point $t_{0}$ ).

Let $M \subset X$. Then by a symbol $l(M)$ (resp. $l_{0}(M)$ ) we will denote a set of paths (resp. closed paths) which lie in $M$ and are correlated with $L_{c}^{*}$.

From these definitions it easily follows that if $\gamma^{\prime}\left(t_{0}\right) \in L_{c}^{*}$ for some $t_{0} \in$ $(\alpha, \beta)$, then $\gamma$ is correlated with $L_{c}^{*}$ at $t_{0}$.

If $p, q \in X$, by the symbol $\tilde{p} q$ we will denote any curve where $p$ and $q$ are the beginning and the end of one respectively.

LEMmA 3.1. If $M \subset X$ is a domain, then for any $p, q \in M$ there exists a path $\tilde{p q} \in l(M)$.

P roof. Such a path can be constructed as in the proof of theorem 2.7.

Therefore we can uniformly approximate any curve lying in the domain by the path correlated with $L_{c}^{*}$.

LEMMA 3.2. If $\gamma$ is correlated with $L_{c}^{*}$ at the point $t_{0} \in(\alpha, \beta)$ and a function $F$ has the lc-derivative at the point $\gamma\left(t_{0}\right)$, then

$$
\left.\frac{d}{d t} c F(\gamma(t))\right|_{t=t_{0}}=\gamma^{\prime}\left(t_{0}\right) D_{\mathrm{lc}} F\left(\gamma\left(t_{0}\right)\right) \text {. }
$$

Proof. For any $a>0$ and sufficiently small $s \neq 0$ we get $\Delta_{s} \gamma:=$ $\gamma\left(t_{0}+s\right)-\gamma\left(t_{0}\right) \in L_{c}^{a}$. Then

$$
c s^{-1}\left[F\left(\gamma\left(t_{0}+s\right)\right)-F\left(\gamma\left(t_{0}\right)\right)\right]=s^{-1} \Delta_{s} \gamma\left(D_{\mathrm{lc}} F\left(\gamma\left(t_{0}\right)\right)+d_{s}\right),
$$

where $d_{s}=A_{c}^{l}\left(\Delta_{s} \gamma\right)\left[F\left(\gamma\left(t_{0}\right)+\Delta_{s} \gamma\right)-F\left(\gamma\left(t_{0}\right)\right)\right]-D_{\mathrm{lc}} F\left(\gamma\left(t_{0}\right)\right)$ for $\Delta_{s} \gamma \neq 0$ and $d_{s}=0$ for $\Delta_{s} \gamma=0$. If $s \rightarrow 0$, then $\Delta_{s} \gamma \rightarrow 0$ and $d_{s} \rightarrow 0$.

Hence we obtain

3.3 TheOREM. If $M \subset X$ is a domain and a continuous function $f$ has the lc-primitive function $F$ on $M$, then for any $p, q \in M$ and any path $\tilde{p q} \in l(M)$

$$
\int_{p^{-} q} f(z) d z=c(F(q)-F(p))
$$




\section{Integral theorems}

Let us now suppose that $X$ is an algebra with unity $e$. Obviously, $e \in L_{e}^{*}$. From 3.3 we have that if $M \subset X$ is a domain and a continuous function $f$ has the le-primitive function $F$ on $M$, then for any $p \in M$

$$
F(x)=\int_{p^{-} x} f(z) d z+F(p)
$$

where $x \in M$ and $\tilde{p x} \in l(M)$.

The following set: $\Delta=\Delta(x, y, z)=\left\{t_{1} x+t_{2} y+t_{3} z: t_{1}+t_{2}+t_{3}=\right.$ $\left.1, t_{1}, t_{2}, t_{3} \geq 0\right\}$ will be called a triangle of with vertices $x, y, z \in X$, $x \neq y \neq z$. A closed path $\gamma$ such that $\gamma^{*}$ is a polygonal line with the vertices $x, y, z$ is called the boundary of the triangle $\Delta$ and will be denoted by $\partial \Delta$.

LemMa 4.1. If the function $f: B \rightarrow X$ is continuous on the ball $B C$ $X$ and $\int_{\partial \Delta} f(z) d z=0$ for a boundary $\partial \Delta \in l_{0}(B)$ of any triangle $\Delta C$ $B$, then $f$ has Fréchet differentiable function which is le-primitive in some neighbourhood of any point of the ball $B$.

Proof. 1) Let $p \in B, K_{p}:=B \cap\left(p+L_{e}^{*}\right)$ and let $F_{p}(x):=\int_{\langle p, x\rangle} f(z) d z$, for each $x \in B$. For any $x \in K_{p}$ there exist $b, r>0$ such $B(x, r) \subset B \cap\left(p+L_{e}^{b}\right)$ (see 1.4). For any $a>0$ let $h \in L_{e}^{a}$ and $\|h\|<r$. Then $\partial \Delta(p, x, x+h) \in l_{0}(B)$. Therefore $h^{-1}\left(F_{p}(x+h)-F_{p}(x)\right)=\int_{0}^{1} f(x+t h) d t$. From this it follows that

$$
\left\|\int_{0}^{1} f(x+t h) d t-f(x)\right\| \leq \sup _{t}\|f(x+t h)-f(x)\| \underset{h \rightarrow 0}{\longrightarrow} 0,
$$

so $D_{\mathrm{le}} F_{p}(x)=f(x)$. This gives that $F_{p}$ is an le-primitive function of $f$ in $K_{p}$.

2) Let us fix $x \in B$. Since $L_{e}^{*}$ is an open set, we can choose $q \in X$ in such a way that $B \subset q+L_{e}^{*}$. $L_{e}^{*}$ has property (*) so, for $t \in \mathbb{R}, t \neq 1$, we have $(x-q) t \in L_{e}^{*}$. There also exists a number $t_{1} \neq 1$ such that $p:=$ $q+t_{1}(x-q) \in B$. Consequently $x \in B \cap\left(p+L_{e}^{*}\right)=K_{p}$. From part 1) $F_{p}$ is the le-primitive function in some neighbourhood $B_{x}$ of the point $x$.

3) We will show that $F_{p}$ has the Fréchet derivative at any point $z \in B_{x}$. Let us fix $z \in B_{x}$, and $h \in X$ such that $z+h \in B_{x}$. By 2.6 there exists such $G>0$ and $y_{h} \in\left(z+L_{e}^{*}\right) \cap\left(z+h+L_{e}^{*}\right)$ that $\left\|z-y_{h}\right\| \leq G\|h\|$. Therefore the paths $\left\langle y_{h}, z+h\right\rangle,\left\langle z, y_{h}\right\rangle$ are correlated with $L_{e}^{*}$. In addition if $\|h\|$ is sufficiently small, then $y_{h} \in B_{x}$.

From the above

$$
\Delta_{h} F_{p}(z):=F_{p}(z+h)-F_{p}(z)=\iint_{\left\langle z, y_{h}\right\rangle} f(\xi) d \xi+\int_{\left\langle y_{h}, z+y_{h}\right\rangle} f(\xi) d \xi .
$$


Taking $\gamma_{1, h}(t)=z+t\left(y_{h}-z\right), \gamma_{2, h}(t)=y_{h}+t\left(z+h-y_{h}\right)$, for $t \in\langle 0,1\rangle$, we obtain

$$
\Delta_{h} F_{p}(z)=\left(y_{h}-z\right) \int_{0}^{1}\left[f\left(\gamma_{2, h}(t)\right)-f\left(\gamma_{1, h}(t)\right)\right] d t+h \int_{0}^{1} f\left(\gamma_{2, h}(t)\right) d t .
$$

Let $R(z, h):=\Delta_{h} F_{p}(z)-h f(z)$. Then

$$
\|R(z, h)\| \leq\|h\|\left(G \sup _{t}\left\|f\left(\gamma_{2, h}(t)\right)-f\left(\gamma_{1, h}(t)\right)\right\|+\sup _{t}\left\|f\left(\gamma_{2, h}(t)\right)-f(z)\right\|\right) .
$$

Thus and by continuity of $f$ it follows that $\lim _{h \rightarrow 0}\|h\|^{-1}\|R(z, h)\|=0$. Hence the mapping $F_{p}^{\prime}(z): h \mapsto h f(z)$ is the Fréchet derivative of a function $F_{p}$ at the point $z$.

From part 3) of the above proof we obtain

4.2 Corollary. Let $M \subset X$ be a domain and let $f$ be continuous on $M$. If $f$ has an le-primitive function $F$ on $M$, then $F \in \mathcal{H}_{\mathrm{le}}(M)$.

Lemma 4.3. Let $B$ be a ball in $X$. If $f \in \mathcal{H}_{\mathrm{le}}(B)$, then for any triangle $\Delta \subset B$ we have $\int_{\partial \Delta} f(z) d z=0$. (We do not assume that $\partial \Delta$ is correlated with $L_{e}^{*}$.)

Proof. In the well known way we can construct a decreasing sequence of triangles $\Delta \supset \Delta_{1} \supset \Delta_{2} \supset$... such that

$$
\left\|\int_{\partial \Delta} f(z) d z\right\| \leq 4^{n}\left\|\int_{\partial \Delta} f(z) d z\right\| \text { and }\left|\partial \Delta_{n}\right|=|\partial \Delta| 2^{-n} .
$$

The compactness of $\Delta$ in $X$ implies the existence of the point $x_{0} \in \bigcap \Delta_{n}$. Since $f$ has Fréchet derivative at $x_{0}$, thus there exists a number $d>0$ and a function $h \mapsto R\left(x_{0}, h\right)$, such that

$$
\begin{aligned}
& f\left(x_{0}+h\right)-f\left(x_{0}\right)=\delta_{x} f(h)+R\left(x_{0}, h\right) \quad \text { for }\|h\|<d, \\
& \text { and } \lim _{h \rightarrow 0}\|h\|^{-1}\left\|R\left(x_{0}, h\right)\right\|=0 .
\end{aligned}
$$

From (2), for $x \in B\left(x_{0}, d\right):=B_{0}$ we get (see 2.2)

$$
f(x)=f\left(x_{0}\right)+\left(x-x_{0}\right) D_{\mathrm{le}} f\left(x_{0}\right)+R\left(x_{0}, x-x_{0}\right) .
$$

Hence for $n \in \mathbb{N}$

$$
\begin{aligned}
\left\|\int_{\partial \Delta} f(x) d x\right\| \leq & \left\|\int_{\partial \Delta} f\left(x_{0}\right) d x\right\|+\left\|\int_{\partial \Delta}\left(x-x_{0}\right) d x D_{\mathrm{le}} f\left(x_{0}\right)\right\| \\
& +\left\|\int_{\partial \Delta} R\left(x_{0}, x-x_{0}\right) d x\right\| .
\end{aligned}
$$


By a simple computation we obtain that the values of the first two components of the last sum are equal to zero. Let us take for $x \in B_{0} g\left(x-x_{0}\right):=$ $\left\|x-x_{0}\right\|^{-1}\left\|R\left(x_{0}, x-x_{0}\right)\right\|$ for $x \neq x_{0}$, and $g(0)=0$.

Since $\left\|x-x_{0}\right\| \leq\left|\partial \Delta_{n}\right|$ for $x \in \partial \Delta_{n}$ so we infer that

$$
\begin{aligned}
\| R\left(x_{0}, x-\right. & \left.x_{0}\right) d x \| \\
& \leq\left|\partial \Delta_{n}\right| \sup \left\{\left\|R\left(x_{0}, x-x_{0}\right)\right\|: x \in \partial \Delta_{n}\right\} \\
& \leq\left|\partial \Delta_{n}\right| \sup \left\{g\left(x-x_{0}\right): x \in \partial \Delta_{n}\right\} \sup \left\{\left\|x-x_{0}\right\|: x \in \partial \Delta_{n}\right\} \\
& \leq 4^{-n}\left|\partial \Delta_{n}\right|^{2} \sup \left\{g\left(x-x_{0}\right): x \in \partial \Delta_{n}\right\} .
\end{aligned}
$$

From (4), (1) and continuity of $g$ at the point zero there holds

$$
\left\|\int_{\partial \Delta} f(z) d z\right\| \leq|\partial \Delta|^{2} \sup \left\{g\left(x-x_{0}\right): x \in \partial \Delta_{n}\right\} \underset{n \rightarrow \infty}{\longrightarrow} 0 .
$$

From 4.1 and 4.3 we conclude

4.4 THEOREM. If $M \subset X$ is an open set and $f \in \mathcal{H}_{\mathrm{le}}(M)$, then $f$ has differentiable in Fréchet sense function le-primitive in a neighbourhood of any point of the set $M$.

Let $M \subset X$ and $\gamma_{0}, \gamma_{1}$ be the paths in $M$ having a common interval of the parameters $I=\langle 0,1\rangle$, common initial and terminal points $z_{0}$ and $z_{1}$, respectively. These paths will be called homotopically equivalent or homotopic in $M$ if there exists a continuous mapping $h: I \times I \rightarrow M$ such that $h(0, t)=\gamma_{0}(t), h(1, t)=\gamma_{1}(t)$ and $h(s, 0)=z_{0}, h(s, 1)=z_{1}$ for $s, t \in I$.

If the paths $\gamma_{0}$ and $\gamma_{1}$ are closed, then they are said to be homotopic in $M$ if $h(s, 0)=h(s, 1)$ for $s \in I$. In both cases we will write: $\gamma_{0} \sim \gamma_{1}$ (in $M$ ).

Using the above results the proof of the following theorem can be obtained in the similar way as the proof of its well-known counterpart from the classical complex analysis.

4.5 Theorem. Let $M$ be a domain in $X$ and $f \in \mathcal{H}_{\mathrm{Ic}}(M)$,

(a) if $\gamma_{0}, \gamma_{1} \in l(M)$ and $\gamma_{0} \gamma_{1}$ (in $\left.M\right)$, then $\int_{\gamma_{0}} f(z) d z=\int_{\gamma_{1}} f(z) d z$;

(b) for any $x \in M$ and for any path $\gamma \in l_{0}(M)$ if $\gamma^{\sim} x$ (in $M$ ), then $\int_{\gamma} f(z) d z=0$ (by $x$ we denote here a constant path);

(c) if $M$ is simply connected, then $\int_{\gamma} f(z) d z=0$ for any path $\gamma \in l_{0}(M)$ and $f$ has le-primitive function $F \in \mathcal{H}_{\mathrm{le}}(M)$.

The proofs of the analogoues theorems of the classical complex analysis (cf. e.g. [5]) are based on the fact of the local existence of the primitive function to the holomorphic function in the domain $M \subset \mathbb{C}$. In view of 4.4. the functions of the class $\mathcal{H}_{l c}(M)$ satisfy that property. 


\section{Surrounding sets}

Let $X$ be an algebra with unity $e$ and $\operatorname{dim} X \geq 2$.

By a $k$-valent circle $(k \in \mathbb{N})$ with a centre at the point 0 we mean a closed curve $\mu$ whose each point, except its common beginning and end, is $k$-valent lying in some sphere and some two-dimensional linear subspace $X$. If $x_{0} \in X$, then a curve $\mu+x_{0}$ is called $k$-valent circle of the center $x_{0}$.

A set $M \subset X$ is called surroundable in $X \backslash M$ if:

(1) $0 \in M, M$ has property (*) and $X \backslash M$ is not simply connected (We treat $X \backslash M$ as the topological space with induced topology);

(2) there exists a circle $\mu$ with a centre at zero such that $\mu \cap M=\emptyset$.

We say that a curve $\gamma$ surrounds set $M$ if $\tilde{\gamma} \mu$ (in $X \backslash M$ ). Then we will write: $\gamma \operatorname{surr} M$. If $\mu$ is a $k$-valent circle then we will say that $\gamma$ surrounds $M k$-valent and we will write $\gamma \operatorname{surr}_{k} M$. For some $D \subset X$ by a symbol $\gamma \operatorname{surr}_{k} M$ (in $D$ ) there will be denoted the fact that $\gamma \operatorname{surr}_{k} M$ and $\gamma^{*} \subset D$.

For any $x \in X$ and surroundable $M$ the notation $\gamma \operatorname{surr}(M+x)($ in $D)$ denotes: $(\gamma-x) \operatorname{surr} M$ (in $D-x)$.

Remark. (a) Obviously, if $M$ is a surroundable set, then there exists a two-dimensional subspace (i.e. a plane) $L$ such that $M \cap L=\{0\}$ (a circle $\mu$ lies in $L$ ).

(b) Let $K=\mathbb{R}, \operatorname{dim} X>2$ and let $L$ be a linear subspace of $X$. Then there exists a linear subspace $L^{c} \subset X$ such that $L \cap L^{c}=\{0\}$ and any element $x \in X$ can be uniquely expressed in the form: $x=x^{l}+x^{c}, x^{l} \in L, x^{c} \in L^{c}$. $L^{c}$ is called complementary to the subspace $L$. A map $P_{L}: X \ni x \mapsto x^{l} \in L$ will be called a projection on $L$ (parallel to $L^{c}$ ). If $P_{L}$ is continuous, then $P_{L}$ is a retraction of the $X \backslash L^{c}$ onto $L \backslash\{0\}$.

Let us assume that $\operatorname{dim} L_{2}=2$. Then $L_{2}^{c}$ is a surroundable set in $X \backslash L_{2}^{c}$. Taking for $\mu$ any circle of the centre in 0 lying in $L_{2}$ we have: $\mu \operatorname{surr} L_{2}^{c}$ (in $L_{2}$ ). Moreover, $\mu$ surr $M$ (in $L_{2}$ ), where $M$ is any union of the complementary subspaces to $L_{2}$. Also if $z \in L_{2}^{c}$ and $\gamma=\mu+z$, then $\gamma \operatorname{surr} L_{2}^{c}$ (in $X \backslash L_{2}^{c}$ ).

Using the Mazur-Gelfand theorem we see that if $X$ is a commutative Banach-algebra over $\mathbb{R}$ with unity $e$, then any maximal ideal $M$ in this algebra is surroundable in $L_{e}^{*}$ if codim $M=2$, and $M$ is not surroundable in $L_{e}^{*}$ if codim $M=1$.

If $X$ is an algebra over $\mathbb{C}$, then every maximal ideal $M$ is surroundable in $L_{e}^{*}$. Taking then any maximal ideal $M$ we have that a plane $L_{2}=\{\lambda e$ : $\lambda \in \mathbb{C}\}$ is complementary to $M$. Every element $x \in X$ can be uniquely represented in the form: $x=\lambda e+x^{M}$, where $x^{M} \in M, \lambda e=x^{\curlyvee}(M) e=P_{L_{2}}(x)$, $\widehat{x}(M)$ is the Gelfand transformation. If $\gamma \operatorname{surr} M$ (in $L_{e}^{*}$ ), then $P_{L_{2}}(\gamma)=$ $\gamma(M) e$ and $\gamma^{-}(M) e \operatorname{surr} M$ (in $\left.L_{2} \backslash\{0\}\right)$.

Let $M \subset X \backslash L_{e}^{*}$ be surroundable in $L_{e}^{*}$ and let $\bar{\gamma}$ be a path in $L_{e}^{*}$ such 
that $\bar{\gamma} \operatorname{surr}_{1} M$. For any closed path $\gamma$ let

$$
S(\bar{\gamma}, M, \gamma):=\left\{z \in X:(\gamma-z)^{\gamma} \bar{\gamma}\left(\text { in } L_{e}^{*}\right)\right\}
$$

Lemma 5.1. (a) $S(\bar{\gamma}, M, \gamma)$ is open. For any $z \in X$ and $t \in \mathbb{R}_{+}$ $t S(\bar{\gamma}, M, \gamma)=S(\bar{\gamma}, M, t \gamma)$ and $z+S(\bar{\gamma}, M, \gamma)=S(\bar{\gamma}, M, \gamma+z)$.

(b) For any ball $B \subset X$ there exists a path $\gamma \in l_{0}(X)$ such that $B \subset$ $S(\bar{\gamma}, M, \gamma)$.

(c) If $D \subset X$ is an open set and $z \in D$, then there exists such $\gamma \in l_{0}(D)$ that $z \in S(\bar{\gamma}, M, \gamma)$.

The above lemma follows promptly from the definition (5.1). It is obvious that for any path $\gamma, \gamma^{*}$ is a compact set. Thus, if $\gamma$ lies in $L_{e}^{*}$, then it lies in $L_{e}^{a}$ for some $a>0$. Let us denote $P_{\gamma}:=\sup \left\{a: \gamma^{*} \subset L_{e}^{a}\right\}$. Then $P_{\gamma}>0$ and $P_{\gamma}=\inf \left\{P_{x}: x \in \gamma^{*}\right\}$ (see p. 1).

\section{Integral formula}

Now let $X$ be a commutative algebra with unity $e$. In this case we will use a short notation: $A_{e}^{l}(z)=z^{-1}$ for $z \in L_{e}^{*}, z^{0}=e, z^{n}=\left(z^{n-1}\right) z$ for $n \in \mathbb{N}$.

Applying 2.5 we can easily prove the following

6.1 THEOREM. If the functions $f$ and $g$ have the le-derivative at some point $x_{0} \in X$, then the function $f g$ has also the le-derivative there and

$$
D_{\mathrm{le}} f g\left(x_{0}\right)=f\left(x_{0}\right) D_{l e} g\left(x_{0}\right)+g\left(x_{0}\right) D_{l e} f\left(x_{0}\right) .
$$

6.2 Theorem. $A_{e}^{l} \in \mathcal{H}_{l e}\left(L_{e}^{*}\right)$. For any $x \in L_{e}^{*} \quad D_{\mathrm{le}} A_{e}^{l}(x)=-\left[A_{e}^{l}(x)\right]^{2}$.

P r oof. Let $x \in L_{e}^{*}$. If $\|h\|$ is sufficiently small, then $x+h \in L_{e}^{*}$ and $A_{e}^{l}(x+h)-A_{e}^{l}(x)=-h A_{e}^{l}(x) A_{e}^{l}(x+h)$. Hence $\delta_{x} A_{e}^{l}(h)=-h\left[A_{e}^{l}(x)\right]^{2}$. For $h \in L_{e}^{*}$ we get $\delta_{x} A_{e}^{l}(h) A_{e}^{l}(h)=-\left[A_{e}^{l}(x)\right]^{2}$. Now it is enough to apply 2.3 and 6.1.

Let $M \subset X \backslash L_{e}^{*}$ be surroundable in $L_{e}^{*}$ and $\bar{\gamma}$ be a path in $L_{e}^{*}$ such that $\bar{\gamma} \operatorname{surr}_{1} M$.

6.3 TheOREM. If $D \subset X$ is a convex domain and $f \in \mathcal{H}_{l e}(D)$, then for any path $\gamma \in l_{0}(D)$ and $z \in D \cap S(\bar{\gamma}, M, \gamma)$

$$
\Pi_{\bar{\gamma}} f(z)=\int_{\gamma} f(\xi)(\xi-z)^{-1} d \xi
$$

where $\Pi_{\bar{\gamma}}=\int_{\bar{\gamma}} \xi^{-1} d \xi$.

Pr o of. Since $\gamma^{*}-z \subset L_{e}^{*}$, then there exists a number $a>0$ such that $\gamma^{*} \subset\left(\right.$ Int $\left.L_{e}^{a}+z\right) \cap D:=D_{a}$. Let us consider a function $g(x):=f(x)(x-z)^{-1}$ for $x \in D_{a}$. From 6.1 and 6.2 we have $g \in \mathcal{H}_{\mathrm{le}}\left(D_{a}\right)$. Let us remark that for 
any $s \in(0,1\rangle \gamma_{s}:=s \gamma+z(1-s) \in l_{0}\left(D_{a}\right)$ and $\gamma_{s} \gamma$ (in $\left.D_{a}\right)$. Also $\gamma_{s}$ and $\gamma$ lies in one component of the set $D_{a} .4 .5$ implies that

$$
\begin{aligned}
I_{\gamma} & :=\int_{\gamma} g(\xi) d \xi=\int_{\gamma_{s}} g(\xi) d \xi \\
& =\int_{\gamma_{s}} f(z)(\xi-z)^{-1} d \xi+\int_{\gamma}[f(\xi)-f(z)](\xi-z)^{-1} d \xi .
\end{aligned}
$$

The last two integrals will be denoted by $I_{1}$ and $I_{2}$. Obviously $I_{1}=$ $I_{\bar{\gamma}} f(z)$. For $\xi \in \gamma_{s}^{*}$ we have $\left\|(\xi-z)^{-1}\right\| \leq a^{-1}\|\xi-z\|^{-1}$ (see (2.1)) and $d:=\left|\gamma_{s}\right| \sup \left\{\|\xi-z\|^{-1}: \xi \in \gamma_{s}^{*}\right\}=|\gamma| \sup \left\{\|\eta-z\|^{-1}: \eta \in \gamma^{*}\right\}<\infty$. Hence and by continuity of $f$ at the point $z$ we get:

$$
\left\|I_{2}\right\| \leq d a^{-1} \sup \left\{\|f(\xi)-f(z)\|: \xi \in \gamma_{s}^{*}\right\} \underset{s \rightarrow 0}{\longrightarrow} 0 .
$$

Therefore $I_{2}=0$.

Using the above result and 5.1 (c) we can prove the following corollary.

6.4 CoRollary. If $D$ is an open set in $X$, then for any $z_{0} \in D$ there exists a path $\gamma \in l_{0}(D)$ and $r>0$ such that equation (6.1) holds for $z \in$ $B\left(z_{0}, r\right)$

\section{Taylor series}

Let us suppose now that $X$ is a Banach commutative algebra with unity $e$. In this case $L=L_{e}^{*}=r_{l}(e):=G$ is the multiplicative group of invertible elements of algebra $X$.

A function $f$ is said to be analytic at the point $z_{0} \in X$ if there exists a ball $B=B\left(z_{0}, r\right)(r>0)$ and a sequence $\left(a_{n}\right)$ of elements of $X$ such that

$$
f(z)=\sum_{n=0}^{\infty} a_{n}\left(z-z_{0}\right)^{n} \quad \text { for } z \in B .
$$

A function $f$ is said to be analytic in set $D \subset X$ if $f$ is analytic at every point of this set. A class of analytic functions in $D$ will be denoted by $A N(D)$.

For $k, n \in \mathbb{N}_{0}$ and $z \in X$ we have $D_{\mathrm{le}}^{k} z^{n}=\frac{n !}{(n-k) !} z^{n-k}$ for $k \leq n$ and $D_{\mathrm{le}}^{k} z^{n}=0$ for $k>n$.

Hence and from the well-known properties of power series we have

7.1 THEOREM. If the function $f$ is a sum of the series (7.1) in certain ball $B\left(z_{0}, r\right)$, then there exists a ball $B_{0}=B\left(z_{0}, r_{1}\right), 0<r_{1} \leq r$, such that $f \in \mathcal{K}_{\mathrm{le}}^{\infty}(B)$ and for any $k \in \mathbb{N}$ and $z \in B$

$$
D_{\mathrm{le}}^{k} f(z)=\sum_{n=k}^{\infty} \frac{n !}{(n-k) !} a_{n}\left(z-z_{0}\right)^{n-k} \text {. }
$$




\section{Moreover}

$$
a_{n}=\frac{1}{n !} D_{\mathrm{le}}^{n} f\left(z_{0}\right) \quad \text { for } n=0,1,2, \ldots
$$

Therefore if $f \in A N(D)$ for $D \subset X$, then $D_{\mathrm{le}}^{k} f \in A N(D)$ for any $k \in \mathbb{N}$ and $A N(D) \subset \mathcal{H}_{\mathrm{le}}^{\infty}(D)$.

Lemma 7.2. Let $\gamma$ be a curve in $X$ and $z_{0} \in X$. If for some $z \in X$

$$
\gamma^{*}-z \subset G, \quad \gamma^{*}-z_{0} \subset G
$$

and

$$
\left\|z-z_{0}\right\|<P_{\gamma-z_{0}} \operatorname{dist}\left(\gamma^{*}, z_{0}\right):=H\left(\gamma, z_{0}\right),
$$

then for each $\xi \in \gamma^{*}$

$$
(\xi-z)^{-1}=\sum_{n=0}^{\infty}\left(z-z_{0}\right)^{n}\left(\xi-z_{0}\right)^{-n-1},
$$

which is uniformly convergent on $\gamma^{*}$.

Proof. If $y, y-x \in G$ and $x y^{-1} \in B(0,1)$, then

$$
(y-x)^{-1}=\sum_{n=0}^{\infty} x^{n} y^{-n-1} .
$$

Obviously, $y-x=y\left(e-x y^{-1}\right)$. Hence

$$
(y-x)^{-1}=y^{-1}\left(e-x y^{-1}\right)^{-1}=\sum_{n=0}^{\infty} x^{n} y^{-n-1} .
$$

For $\xi \in \gamma^{*}$ we have:

$$
\left\|\left(z-z_{0}\right)\left(\xi-z_{0}\right)^{-1}\right\| \leq \frac{\left\|z-z_{0}\right\|}{P_{\gamma-z_{0}}\left\|\xi-z_{0}\right\|}<\frac{\operatorname{dist}\left(\gamma^{*}, z_{0}\right)}{\left\|\xi-z_{0}\right\|} \leq 1 .
$$

Putting $x=z-z_{0}$ and $y=\xi-z_{0}$ in (4) we obtain (3). For fixed $z$ there exists $t \in(0,1)$ such that $\left\|z-z_{0}\right\|=t H(\gamma, z)$. Denoting by $C_{n}(\xi)$ the $n$-th term of series (3) we have

$$
\left\|C_{n}(\xi)\right\| \leq\left\|\left(z-z_{0}\right)^{n}\right\|\left\|\left[\left(\xi-z_{0}\right)^{-1}\right]^{n+1}\right\| \leq P_{\gamma-z_{0}}^{-n-1} \frac{\left\|z-z_{0}\right\|^{n}}{\left\|\xi-z_{0}\right\|^{n+1}} .
$$

Hence

$$
\lim \sup \sqrt[n]{\left\|C_{n}(\xi)\right\|} \leq P_{\gamma-z_{0}}^{-1} \frac{\left\|z-z_{0}\right\|}{\left\|\xi-z_{0}\right\|} \leq t<1 \quad \text { for any } \xi \in \gamma^{*} .
$$

This completes the prof of uniformly convergence of series (3) on $\gamma^{*}$.

A class of such commutative algebras $X$ with unity $e$ for which there exists a set $M \subset X \backslash G$ surroundable in $G$ will be denoted by $(S)$. The $\left(S_{0}\right)$ 
denotes a subset of the class $(S)$ including such algebras $X$ for which there exists $M \subset X \backslash G$ and $\gamma \in l_{0}(G)$ such that $\gamma$ surr $M$ and $\Pi_{\gamma}=\int_{\gamma} \xi^{-1} d \xi \in G$.

We can prove that if $X \in\left(S_{0}\right)$, then the function $z \mapsto \exp z$ has a period $\omega \in G$.

Examples. In examples 2.8 (b), (d), (f) the algebras $X$ are of the class $\left(S_{0}\right)$ but in the (c) and (e) $X \notin(S)$. From remarks in the chapter 5 , if $X$ is an algebra over $\mathbb{C}$, then $X \in(S)$.

Let us now suppose that $M \subset X \backslash G$ is a surroundable set in $G$ and let us fix a path $\bar{\gamma} \in l_{0}(G)$ such that $\bar{\gamma} \operatorname{surr}_{1} M$. Applying Lemma 7.2 and Theorem 6.3 we easily obtain

7.3 Theorem. Let $D \subset X$ be a convex domain, $\gamma \in l_{0}(D), z_{0} \in$ $S(\bar{\gamma}, M, \gamma)$ and $f \in \mathcal{H}_{\mathrm{le}}(D)$. Then

(7.2) $\Pi_{\bar{\gamma}} f(z)=\sum_{n=0}^{\infty} b_{n}\left(z-z_{0}\right)^{n} \quad$ for $z \in D \cap S(\bar{\gamma}, M, \gamma) \cap B\left(z_{0}, H\left(\gamma, z_{0}\right)\right)$, where

$$
b_{n}=\int_{\gamma} f(\xi)\left(\xi-z_{0}\right)^{-n-1} d \xi \quad \text { for } n \in \mathbb{N}_{0}, \text { and } \Pi_{\bar{\gamma}}=\int_{\bar{\gamma}} \xi^{-1} d \xi .
$$

Moreover for $n \in \mathbb{N}_{0}$

$$
\left\|b_{n}\right\| \leq|\gamma| \frac{\sup \left\{\|f(\xi)\|: \xi \in \gamma^{*}\right\}}{P_{\gamma-z_{0}}^{n+1} \operatorname{dist}\left(\gamma^{*}, z_{0}\right)^{n+1}} .
$$

Remark. Theorem 7.1 is equivalent to the Taylor theorem and inequalities (7.3) are analogues to Cauchy ones.

From Theorem 7.3 and Lemma 5.1 we obtain

7.4 Corollary. If $X \in(S), D \subset X$ is an open set and $f \in \mathcal{H}_{\mathrm{le}}(D)$, then $\Pi_{\bar{\gamma}} f \in A N(D)$.

7.5 Corollary. If $X \in\left(S_{0}\right)$ and $D \subset X$ is an open set, then

$$
\mathcal{H}_{\text {le }}(D)=A N_{\text {le }}(D)=\mathcal{H}_{\text {le }}^{\infty}(D) \text {. }
$$

Obviously, 7.4 implies that $\mathcal{H}_{\text {le }}(D) \subset A N(D)$ and from 7.1 we have $A N(D) \subset \mathcal{H}_{\mathrm{le}}^{\infty}(D)$.

From 7.3 and 7.1 we have

7.6 Corollary. Let us suppose that $X \in(S), D \subset X$ is a convex domain $\gamma \in l_{0}(D)$ and $f \in \mathcal{H}_{\mathrm{le}}(D)$. If $z_{0} \in D \cap S(\bar{\gamma}, M, \gamma)$, then for any $n \in \mathbb{N}_{0}$

$$
D_{\text {le }}^{n}\left[\Pi_{\bar{\gamma}} f\right]\left(z_{0}\right)=n ! \int_{\gamma} f(\xi)\left(\xi-z_{0}\right)^{-n-1} d \xi
$$


and there exists a neighbourhood $U$ of the point $z_{0}$ in which

$$
\Pi_{\bar{\gamma}} f(z)=\sum_{n=0}^{\infty} \frac{1}{n !} D_{\mathrm{le}}^{n}\left[I_{\bar{\gamma}} f\right]\left(z_{0}\right)\left(z-z_{0}\right)^{-n-1} .
$$

In particular, if $\Pi_{\bar{\gamma}} \in G$ (i.e. $X \in\left(S_{0}\right)$ ), then

$$
f(z)=\sum_{n=0}^{\infty} \frac{1}{n !} D_{\mathrm{le}}^{n} f\left(z_{0}\right)\left(z-z_{0}\right)^{n} \quad \text { for } z \in U .
$$

7.8 Theorem. (Morera). Let $X \in\left(S_{0}\right)$. If $f$ is a continuous function on the open set $D \subset X$ and $\int_{\partial \Delta} f(z) d z=0$ on the boundary $\partial \Delta \in l_{0}(D)$ of any triangle $\Delta \subset D$, then $f \in \mathcal{H}_{\mathrm{le}}(D)$.

Proof. Let us fix $z_{0} \in D$. From 4.1 it follows that $f$ has the Fréchet differentiable le-primitive function $F$ in some neighbourhood $U$ of the point $z_{0}$. Since $F \in \mathcal{H}_{\mathrm{le}}(U)$, so $F \in A N(U)$ and $f=D_{\mathrm{le}} F \in \mathcal{H}_{\mathrm{le}}(U)$.

Using the obtained results the proof of the following theorem is analogous to the proof of Liouville theorem.

7.9 ThEOREM. (Liouville). If $X \in\left(S_{0}\right), f \in \mathcal{H}_{\mathrm{le}}(X)$ and $f$ is bounded on $X$, then $f$ is constant.

\section{Laurent series}

Let $X$ be a Banach commutative algebra with unity $e$, and let $G$ be the set of invertible elements of algebra $X$.

The Laurent series of the center $z_{0} \in X$ will be called the sum of the following series

$$
\sum_{n=1}^{\infty} a_{-n}\left(z-z_{0}\right)^{-n}+\sum_{n=0}^{\infty} a_{n}\left(z-z_{0}\right)^{n}:=\sum_{n=-\infty}^{\infty} a_{n}\left(z-z_{0}\right)^{n},
$$

where $a_{n}(n=0, \pm 1, \pm 2, \ldots)$ are fixed elements of the space $X$.

For any curve $\gamma:\langle a, b\rangle \rightarrow X$ let $\|\gamma\|_{c}:=\sup \{\|\gamma(t)\|: t \in\langle a, b\rangle\}$. Similarly as in Lemma 7.2 we can prove the following

Lemma 8.1. Let $\gamma$ be a curve in $X$ and $z_{0} \in X$. If for some $z \in X$ we have: $\gamma^{*}-z \subset G, z-z_{0} \in G$ and $\left\|z-z_{0}\right\| P_{z-z_{0}}>\left\|\gamma-z_{0}\right\|_{c}$, then for $\xi \in \gamma^{*}$

$$
-(\xi-z)^{-1}=\sum_{n=0}^{\infty}\left(\xi-z_{0}\right)^{n}\left(z-z_{0}\right)^{-n-1}
$$

and this series is uniformly convergent on $\gamma^{*}$.

Let us assume that $X \in(S)$, a set $G$ is connected, $M \subset X \backslash G$ is the sum of all sets surroundable in $G$ and $M$ is also surroundable in $G$. Let us fix $\bar{\gamma} \in l_{0}(G)$ such that $\bar{\gamma} \operatorname{surr}_{1} M$ (in $G$ ). 
Applying Lemmas 8.1, 7.2 and Theorem 6.3 we obtain

8.2 THEOREM. If $X \in(S)$, the sel $G$ is connected, for some $z_{0} \in X$ and $r>0 f \in \mathcal{H}_{\mathrm{le}}\left(K_{0}\left(z_{0}, r\right)\right)$, where $K_{0}\left(z_{0}, r\right)=B\left(z_{0}, r\right) \cap\left(G+z_{0}\right)$, then for any path $\gamma \in l_{0}\left(K_{0}\left(z_{0}, r\right)\right)$ such that $\gamma \operatorname{surr}_{1}\left(M+z_{0}\right)$ we have

$$
\Pi_{\bar{\gamma}} f(z)=\sum_{n=-\infty}^{\infty}\left(z-z_{0}\right)^{n} \int_{\gamma} f(\xi)\left(\xi-z_{0}\right)^{-n-1} d \xi
$$

for $z \in K_{0}\left(z_{0}, r\right) \cap S(\bar{\gamma}, M, \gamma) \cap B\left(z_{0}, H\left(\gamma, z_{0}\right)\right)$. Therefore a function $\Pi_{\bar{\gamma}} f$ is the sum of the Laurent series in some set $\kappa_{0}\left(z_{0}, r_{1}\right), r_{1}>0$.

Remark. 1. We now assume that the set $G$ is not connected. For a certain set $A$ and $\alpha \in A$ let $G_{\alpha}$ denote a component of the set $G, G=$ $\bigcup_{\alpha \in A} G_{\alpha}$ and $M_{\alpha}$ is the sum of all sets surroundable in $G_{\alpha}$. For a certain $\alpha \in A$ let $\bar{\gamma}_{\alpha} \in l_{0}\left(G_{\alpha}\right)$ and $\bar{\gamma}_{\alpha} \operatorname{surr}_{1} M_{\alpha}$ (in $G_{\alpha}$ ). Moreover for $z \in X$ and $r>0$ we denote $K_{0}^{\alpha}(z, r)=B(z, r) \cap\left(G_{\alpha}+z\right)$. We can prove that if $f \in \mathcal{H}_{\mathrm{le}}\left(K_{0}^{\alpha}\left(z_{0}, r\right)\right), \gamma \in l_{0}\left(K_{0}^{\alpha}\left(z_{0}, r\right)\right)$ and $\gamma \operatorname{surr}_{1}\left(M_{\alpha}+z_{0}\right)$ for some $z_{0} \in X$ and $r>0$, then for a certain $r_{1}>0$ and any $z \in K_{0}^{\alpha}\left(z_{0}, r_{1}\right)$ we have:

$$
\Pi_{\bar{\gamma}_{\alpha}} f(z)=\sum_{n=-\infty}^{\infty}\left(z-z_{0}\right)^{n} \int_{\gamma} f(\xi)\left(\xi-z_{0}\right)^{-n-1} d \xi+\Phi(z),
$$

where

$$
\Phi(z)=\int_{\gamma_{2}} f(\xi)(\xi-z)^{-1} d \xi-\int_{\gamma_{1}} f(\xi)(\xi-z)^{-1} d \xi
$$

and $\gamma_{1}, \gamma_{2}$ are some curves satisfying the following relations:

$\gamma_{1} \operatorname{surr}_{1}\left(M_{\alpha}+z_{0}\right)$ (in $\left.z_{0}+G_{\alpha}\right)$ and $\gamma_{2} \operatorname{surr}_{1}\left(M_{\alpha}+z_{0}\right)\left(\right.$ in $\left.z_{0}-G_{\alpha}\right)$.

2. From Theorem 2.2 it follows that if $X$ is a commutative algebra with unity $e, D \subset X$ is an open set and $f \in \mathcal{H}_{\mathrm{le}}(D)$, then for any $z \in D D_{\mathrm{le}} f(z)$ is the derivative of the function $f$ at the point $z$ in the Lorch sense ([3]).

3. For any $x \in X$ we can analogously define the rc-inverse element $y$ (right-hand-c-inverse; see page 2) and consequently we obtain rc-derivative and the $r$-integral. In this way we have an equivalent of Theorem 4.5.

\section{References}

[1] E. Hille, Functional analysis and semi-groups, Colloq. Amer. Math. Soc. 1948.

[2] S. Dineen, Complex Analysis in Locally Convex Spaces, North-Holland Math. Studies, $57,1981$.

[3] E. R. Lorch, The theory of analytic functions in normed abelian vector rings, Trans. Amer. Math. Soc. 54. 1943. 
[4] G. E. Ladas and V. Lakshmikanth am, Differential Equations in Abstract Spaces, Mathematics in Science and Engineering, Academic Press, University of Southern California, New York and London, 1972.

[5] Б. В. III абат, Веедение в комплексмий амализ, Издательство ,Наука”, Москва, 1969.

INTITUTE OF MATHEMATICS

TECHNICAL UNIVERSITY OF LÓDŹ

Al. Politechniki 11

90-924 ŁÓDZ, POLAND

Received October 12, 1993; revised version November 2-nd, 1994, 